\title{
SINGULAR PERTURBATION FOR THE DIRICHLET BOUNDARY CONTROL OF ELLIPTIC PROBLEMS
}

\author{
Faker Ben Belgacem ${ }^{1}$, Henda El Fekih ${ }^{2}$ and Hejer Metoui ${ }^{3}$
}

\begin{abstract}
A current procedure that takes into account the Dirichlet boundary condition with nonsmooth data is to change it into a Robin type condition by introducing a penalization term; a major effect of this procedure is an easy implementation of the boundary condition. In this work, we deal with an optimal control problem where the control variable is the Dirichlet data. We describe the Robin penalization, and we bound the gap between the penalized and the non-penalized boundary controls for the small penalization parameter. Some numerical results are reported on to highlight the reliability of such an approach.
\end{abstract}

Mathematics Subject Classification. 49N05, 49N10, 34D15.

Received: September 10, 2002. Revised: May 21, 2003.

\section{INTRODUCTION}

Most often, the computing codes for elliptic partial differential equations, based on variational numerical methods such as finite elements, take into account the Dirichlet boundary condition using penalization; either by rustic algebraic operations or by adopting the Robin penalization. In addition to an easy treatment of the Dirichlet condition, an essential motivation of the Robin penalization procedure is that it handles discontinuous data relevantly because of the regularity effect induced on the solution. A compulsory question is then: how does this penalization affect the accuracy of the discrete solution? Some mathematical works have arisen out of this question, such as $[3,4,6,8,15,16]$ in which the different authors successfully state the expected convergence rates, and [10] where a sharp asymptotic expansion of the penalized solution with arbitrary order is proven.

For the Dirichlet boundary control of second order equations, where the boundary control variable is generally looked for in $L^{2}$ (the Lebesgue space of the square integrable functions defined on the boundary), the necessity of the Robin penalization is even increased for both practical and theoretical reasons (see $[2,9,13,14,19]$ ). The optimal conditions of the penalized problem yield a mixed problem with the optimal and adjoint states as independent unknowns, set on the standard space $H^{1} \times H^{1}$, (the Sobolev space of the square integrable functions together with their first order derivatives), while the mixed problem coming from the non-penalized equations has to be studied in $H^{2} \times L^{2},\left(H^{2}\right.$ is the space of the square integrable functions together with its first and

Keywords and phrases. Boundary control problems, non-smooth Dirichlet condition, Robin penalization, singularly perturbed problem.

1 Mathématiques pour l'Industrie et la Physique, UMR 5640, Université Paul Sabatier, 31062 Toulouse Cedex 04, France. e-mail: belgacem@mip.ups-tlse.fr

${ }^{2}$ LAMSIN, École Nationale d'Ingénieurs de Tunis, BP 37, 1002 Tunis-Belvédère, Tunisie. e-mail: henda.elfekih@enit.rnu.tn

${ }^{3}$ LAMSIN, École Nationale d'Ingénieurs de Tunis, BP 37, 1002 Tunis-Belvédère, Tunisie. e-mail: hajer.metoui@enit.rnu.tn 
second order derivatives). Both of them can be viewed as the variational formulations of fourth order partial differential equations. The common feeling inclines us rather to handle the weak problem set on $H^{1} \times H^{1}$ which is more attractive especially when approximation is aimed. In addition, the optimal control is expressed by the trace of the adjoint state when Robin penalization is used, instead of its normal derivative in the non-penalized case. These two points are welcomed in view of numerically solving the control problem by variational methods, for instance, the Lagrange finite elements can be naturally used to compute the penalized optimal and adjoint states; then the optimal control is obtained easily as indicated above.

This paper deals with the theoretical and numerical issues of the Robin penalization approach for the boundary control problem and its structure is as follows: Section 2 presents the boundary control problem of the Poisson equation, and particularly a non-standard variational framework that allows for non-smooth Dirichlet data. Next, the optimality conditions are stated using the adjoint state, and the resulting mixed problem is analyzed through the Brezzi-Fortin saddle point theory. The end of the section is devoted to some comments on the regularity of the optimal solution in the case of polygonal domains. In Section 3, the Poisson problem is regularized by a Robin boundary condition and we study the corresponding control problem to establish the existence and stability results. Section 4 is the core of this work, it is addressing the convergence analysis of the penalized optimal solution toward the optimal non-penalized solution, first regarding regular domains where we prove a linear convergence with respect to the penalization parameter, then for polygonal domains for which quasi-optimal results are given. In Section 5, we discuss some numerical tests that support the efficiency of the penalization approach.

Notations - Let $\Omega \subset \mathbb{R}^{2}$ be a bounded domain with a Lipschitz boundary $\Gamma$, the generic point of $\Omega$ is denoted $\boldsymbol{x}=(x, y)$. The Lebesgue space $L^{2}(\Omega)$ is endowed with the norm

$$
\|\psi\|_{L^{2}(\Omega)}=\left(\int_{\Omega}|\psi(\boldsymbol{x})|^{2} \mathrm{~d} \boldsymbol{x}\right)^{\frac{1}{2}} .
$$

The Sobolev space $H^{m}(\Omega), m \geq 1$ is defined in a standard way and provided with the norm

$$
\|\psi\|_{H^{m}(\Omega)}=\left(\sum_{0 \leq|\alpha| \leq m}\left\|\partial^{\alpha} \psi\right\|_{L^{2}(\Omega)}^{2}\right)^{\frac{1}{2}}
$$

where $\alpha=\left(\alpha_{x}, \alpha_{y}\right)$ is a multi-index in $\mathbb{N}^{2}$ and $\partial^{\alpha}=\partial_{x}^{\alpha_{x}} \partial_{y}^{\alpha_{y}}$. The fractional order Sobolev space $H^{\nu}(\Omega), \nu \in$ $\mathbb{R}_{+} \backslash \mathbb{N}$, is defined by the norm

$$
\|\psi\|_{H^{\nu}(\Omega)}=\left(\|\psi\|_{H^{m}(\Omega)}^{2}+\sum_{|\alpha|=m} \int_{\Omega} \int_{\Omega} \frac{\left(\partial^{\alpha} \psi(\boldsymbol{x})-\partial^{\alpha} \psi\left(\boldsymbol{x}^{\prime}\right)\right)^{2}}{\left|\boldsymbol{x}-\boldsymbol{x}^{\prime}\right|^{2+2 \theta}} \mathrm{d} \boldsymbol{x} \mathrm{d} \boldsymbol{x}^{\prime}\right)^{\frac{1}{2}}
$$

where $\nu=m+\theta, m$ is the integer part of $\nu$ and $\theta \in] 0,1[$ its decimal part (see $[1,12,18])$. The closure in $H^{\nu}(\Omega)$ of $\mathscr{D}(\Omega)$ is denoted $H_{0}^{\nu}(\Omega)$, where $\mathscr{D}(\Omega)$ is the space of infinitely differentiable functions whose supports are contained in $\Omega$. For any $\nu>0$, the Hilbert space $H^{\nu}(\Gamma)$ is defined as the range of $H^{\nu+\frac{1}{2}}(\Omega)$ by the trace operator, it is then endowed by the image norm

$$
\|\chi\|_{H^{\nu}(\Gamma)}=\inf _{\psi \in H^{\nu+\frac{1}{2}}(\Omega) ; \psi_{\mid \Gamma}=\chi}\|\psi\|_{H^{\nu+\frac{1}{2}}(\Omega)} .
$$

\section{The Dirichlet Boundary CONTROL PROBlem}

We deal with the optimal control problem where the control variable is the Dirichlet boundary data; the goal consists of choosing the appropriate boundary condition in the state equation (a linear partial differential 
equation for our purpose) so that the solution reaches, or comes close to, a predefined profile. The mathematical model we are interested in can be roughly expressed as follows

$$
\begin{gathered}
\min \quad J(y, u) \\
\left\{\begin{aligned}
-\Delta y=f, & \text { in } \Omega \\
y=u, & \text { on } \Gamma
\end{aligned}\right.
\end{gathered}
$$

where $J(.,$.$) is a given objective function assumed to be sufficiently smooth. The aim we have in mind is to$ specify the minimum when the variable $u$ describes an admissible space. The technical point for studying this optimization problem is then the determination of the appropriate space for $u$ easy to handle and that yields the well pozedness of (1). One might be tempted to work in $H^{\frac{1}{2}}(\Gamma)$, which appears as a natural choice. However, for non-smooth boundary $\Gamma$, such as when $\Omega$ is a polygonal domain, the problem (1) might become ill posed due to a lack of existence results (the explanation of such a fact is given later in Rem. 2.4). The alternative is to resort to the space $L^{2}(\Gamma)$, which although non-standard, provides the functional framework in which the well pozedness of the control problem is guaranteed for general geometries.

Before handling the problem (1) itself, it is necessary to set the appropriate variational formulation of the Poisson-Dirichlet equation when $u \in L^{2}(\Gamma)$. We assume that $f \in L^{2}(\Omega)$, a function $y \in L^{2}(\Omega)$ is said to be a weak solution of the Poisson-Dirichlet problem if

$$
\int_{\Omega} y(-\Delta z) \mathrm{d} \boldsymbol{x}=\int_{\Omega} f z \mathrm{~d} \boldsymbol{x}-\int_{\Gamma} u\left(\partial_{\boldsymbol{n}} z\right) \mathrm{d} \Gamma, \quad \forall z \in D(\Delta),
$$

where $D(\Delta)$ is defined as

$$
D(\Delta)=\left\{z \in H_{0}^{1}(\Omega), \quad \Delta z \in L^{2}(\Omega)\right\}
$$

For the Lipschitz domain $\Omega$, the normal derivative $\left(\partial_{\boldsymbol{n}} z\right)$ makes sense in $L^{2}(\Gamma)$ for each function $z \in D(\Delta)$ (see [12]). - Notice that the norm $\|z\|_{D(\Delta)}:=\|\Delta z\|_{L^{2}(\Omega)}$ is equivalent to the graph norm of $D(\Delta)$ (defined by $\left.\|z\|_{H^{1}(\Omega)}+\|\Delta z\|_{L^{2}(\Omega)}\right)$.

Recalling the fact that the operator $(-\Delta)$ sets an isomorphism from $D(\Delta)$ into $L^{2}(\Omega)$, it is worth making the variable change $y=-\Delta w ; w \in D(\Delta)$. Problem (2) is then reformulated in the following terms: find $w \in D(\Delta)$ such that

$$
\int_{\Omega} \Delta w \Delta z \mathrm{~d} \boldsymbol{x}=\int_{\Omega} f z \mathrm{~d} \boldsymbol{x}-\int_{\Gamma} u\left(\partial_{\boldsymbol{n}} z\right) \mathrm{d} \Gamma, \quad \forall z \in D(\Delta) .
$$

Existence, uniqueness and stability results for problem (2) are proven in a straightforward way by Lax-Milgram Lemma. Moreover, by the interpolation theory of continuous linear operators in Hilbert space, it can be shown that actually $y$ belongs to $H^{\sigma}(\Omega), \forall \sigma<\frac{1}{2}$ with

$$
\|y\|_{H^{\sigma}(\Omega)} \leq C(\sigma)\left(\|f\|_{L^{2}(\Omega)}+\|u\|_{L^{2}(\Gamma)}\right) .
$$

The limit case $\sigma=\frac{1}{2}$ is reached when the boundary $\Gamma$ is regular enough (e.g. of class $\left.\mathscr{C}^{2}\right)$.

Remark 2.1. More regularity on $u\left(\in H^{\frac{1}{2}}(\Gamma)\right)$ allows usage of the standard variational setting that consists of finding $y \in H^{1}(\Omega) ; y_{\mid \Gamma}=u$ such that

$$
\int_{\Omega} \nabla y \nabla z \mathrm{~d} \boldsymbol{x}=\int_{\Omega} f z \mathrm{~d} \boldsymbol{x}, \quad \forall z \in H_{0}^{1}(\Omega) .
$$

Applying Green's formula which states that the unique solution $y$ of (4) verifies as well the weak problem (2) is an easy matter. The $L^{2}$-variational formulation appears then as a natural extension of the $H^{1}$-formulation. 
Remark 2.2. For the approximation of the solution $y$ by applying standard numerical methods, such as finite or spectral element methods, the variational formulation (2) is of no practical use. Designing a numerical method well adapted to that formulation would be cumbersome. A possible process to overcome this difficulty is provided by the Robin penalization of the Dirichlet condition.

Returning to the control problem (1), the optimal control $\bar{u}$ and the optimal state $\bar{y}$ are identified through the optimality conditions. Since no specific technicalities are due to the distributed datum $f$, and to focus only on the difficulties inherent in the boundary control, we make $f=0$, which does not restrict the generality. In many practical configurations the objective function $J$ is quadratic with respect to the state $y$ and to the control $u$;

$$
J(y, u)=\frac{1}{2} \int_{\Omega}\left(y-y_{d}\right)^{2} \mathrm{~d} \boldsymbol{x}+\frac{\beta}{2} \int_{\Gamma} u^{2} \mathrm{~d} \Gamma,
$$

where $y_{d} \in L^{2}(\Omega)$ is a fixed profile and $\beta>0$ is a parameter that can be modulated according to the ratio (approximation quality/energy cost); for instance, high values of $\beta$ express a willingness of energy economy. For the sake of simplicity, we choose $\beta=1$. In the optimal control problem given in (1) the variable $u$ should be taken in $L^{2}(\Gamma)$ and $y \in L^{2}(\Omega)$ is the unique solution of the Poisson-Dirichlet problem in the sense of (2).

\subsection{The optimality conditions}

The minimization problem is convex and coercive, thus it has only one solution $\bar{u} \in L^{2}(\Gamma)$ that can be obtained from the system of optimal conditions. Solve the saddle point problem on the optimal state $\bar{y} \in L^{2}(\Omega)$ and the adjoint state $\bar{p} \in D(\Delta)$ (see $[17])$,

$$
\begin{array}{rlrl}
\int_{\Omega} \bar{y} z \mathrm{~d} \boldsymbol{x}+\int_{\Omega} z(-\Delta \bar{p}) \mathrm{d} \boldsymbol{x} & =\int_{\Omega} y_{d} z \mathrm{~d} \boldsymbol{x}, \forall z \in L^{2}(\Omega), \\
\int_{\Omega} \bar{y}(-\Delta q) \mathrm{d} \boldsymbol{x}-\int_{\Gamma}\left(\partial_{\boldsymbol{n}} \bar{p}\right)\left(\partial_{\boldsymbol{n}} q\right) \mathrm{d} \Gamma & =0, & \forall q \in D(\Delta),
\end{array}
$$

and then take $\bar{u}=-\partial_{\boldsymbol{n}} \bar{p} \in L^{2}(\Gamma)$.

Lemma 2.3. Let $y_{d} \in L^{2}(\Omega)$, then the saddle point problem $(5-6)$ has only one solution $(\bar{y}, \bar{p}) \in L^{2}(\Omega) \times D(\Delta)$ such that

$$
\max \left(\|\bar{p}\|_{D(\Delta)},\|\bar{y}\|_{L^{2}(\Omega)}\right) \leq\left\|y_{d}\right\|_{L^{2}(\Omega)} .
$$

Moreover, the optimal control $\bar{u}$ satisfies

$$
\|\bar{u}\|_{L^{2}(\Gamma)} \leq \frac{1}{\sqrt{2}}\left\|\bar{y}_{d}\right\|_{L^{2}(\Omega)} .
$$

Proof. Among the existence and uniqueness results developed in the Brezzi and Fortin's book [7, Chap. 2] for different types of abstract saddle point problems, we need that addressing non-fully-coercive problems, given in Theorem 2.1 (and completed by comments of p. 49). To apply that theory to our purpose, we first check that the bilinear forms defined on $L^{2}(\Omega) \times L^{2}(\Omega)$ in $(5)$ and on $D(\Delta) \times D(\Delta)$ in $(6)$ are positive definite and semi-definite respectively, and the mixed form defined on $L^{2}(\Omega) \times D(\Delta)$ (involved both in (5) and in (6)) satisfies the inf-sup condition

$$
\inf _{q \in D(\Delta)} \sup _{z \in L^{2}(\Omega)} \frac{\int_{\Omega} z(-\Delta q) \mathrm{d} \boldsymbol{x}}{\|q\|_{D(\Delta)}\|z\|_{L^{2}(\Omega)}}=1
$$

and that

$$
\left\{q \in D(\Delta), \quad \int_{\Omega} z(-\Delta q) \mathrm{d} \boldsymbol{x}=0, \quad \forall z \in L^{2}(\Omega)\right\}=\{0\}
$$


This yields existence and uniqueness of $(\bar{y}, \bar{p})$. For the stability, choose $z=\bar{y}$ in (5) and $q=\bar{p}$ in $(6)$ and summing up we obtain

$$
\|\bar{y}\|_{L^{2}(\Omega)}^{2}+\left\|\partial_{\boldsymbol{n}} \bar{p}\right\|_{L^{2}(\Gamma)}^{2}=\left(y_{d}, \bar{y}\right)_{L^{2}(\Omega)}
$$

which gives the boundedness for $\bar{y}$ and $\bar{u}$. To achieve the bound for $\bar{p}$ take $z=\Delta \bar{p}$ in (5).

Another way to establish the results of Lemma 2.3 without using the saddle point theory is to eliminate $\bar{y}$ in equation (5) and to derive a fourth order variational equation on the adjoint state $\bar{p}$, which is formulated as follows: find $\bar{p} \in D(\Delta)$ such that

$$
\int_{\Omega} \Delta \bar{p} \Delta q \mathrm{~d} \boldsymbol{x}+\int_{\Gamma}\left(\partial_{\boldsymbol{n}} \bar{p}\right)\left(\partial_{\boldsymbol{n}} q\right) \mathrm{d} \Gamma=\int_{\Omega} y_{d}(-\Delta q) \mathrm{d} \boldsymbol{x}, \quad \forall q \in D(\Delta) .
$$

Existence, uniqueness and stability are guaranteed by the coerciveness of the problem. The optimal state is then obtained as $\bar{y}=y_{d}+\Delta \bar{p} \in L^{2}(\Omega)$ and it is easily seen that $(\bar{y}, \bar{p})$ is also the solution of the problem (5-6).

Remark 2.4. The mixed variational problem (5-6) can be expressed under a strong form that reads as follows: find $(\bar{y}, \bar{p}) \in L^{2}(\Omega) \times D(\Delta)$ such that

$$
\begin{aligned}
\bar{y}-\Delta \bar{p} & =y_{d} & & \text { in } \Omega \\
-\Delta \bar{y} & =0 & & \text { in } \Omega \\
\bar{p} & =0 & & \text { on } \Gamma \\
\bar{y} & =-\partial_{\boldsymbol{n}} \bar{p} & & \text { on } \Gamma .
\end{aligned}
$$

Notice that, when $\Omega$ is not sufficiently smooth, like polygons, the optimal control $\bar{u}=-\partial_{\boldsymbol{n}} \bar{p}$ does not belong to $H^{\frac{1}{2}}(\Gamma)$. This indicates that the optimization problem (1) has no solution $\bar{u}$ in $H^{\frac{1}{2}}(\Gamma)$, although the infimum of the cost function exists.

\subsection{Regularity discussion}

The performances of the penalization procedure depend on the regularity of the solution of the mixed problem (5-6). The interaction between the penalization parameter and the corners is governed by the singularities occuring on the optimal and the adjoint states $(\bar{y}, \bar{p})$. We choose, then, to highlight the principal singular parts of them. For our purpose the singularity on the adjoint state is defined to be the part of the expansion of $\bar{p}$ that does not belong to $H^{2}(\Omega)$, while the singular part of the optimal state stands for the component of the expansion of $\bar{y}$ that is not in $H^{1}(\Omega)$.

The well-known regularity results (see $[11,12])$ tell that when the boundary $\Gamma$ is sufficiently smooth $(e . g$. of class $\mathscr{C}^{2}$ ), the functional space $D(\Delta)$ coincides with the standard Sobolev space $H^{2}(\Omega) \cap H_{0}^{1}(\Omega)$. Hence, the adjoint state $\bar{p}$ lays in $H^{2}(\Omega)$, by the trace theorem the control $\bar{u}=-\partial_{\boldsymbol{n}} \bar{p}$ is in $H^{\frac{1}{2}}(\Gamma)$ and then the solution $(\bar{y}, \bar{p})$ of (5-6) belongs to $H^{1}(\Omega) \times\left(H^{2}(\Omega) \cap H_{0}^{1}(\Omega)\right)$ and

$$
\|\bar{p}\|_{H^{2}(\Omega)}+\|\bar{y}\|_{H^{1}(\Omega)} \leq C\left\|y_{d}\right\|_{L^{2}(\Omega)} .
$$

For convex polygonal domains, $\bar{p}$ remains in $H^{2}(\Omega) \cap H_{0}^{1}(\Omega)$ (see $[11,12]$ ), however the regularity on the optimal state $\bar{y}$ is altered. Indeed, because of the angular points (the corners) the normal derivative $\partial_{\boldsymbol{n}} \bar{p}$ can not be in $H^{\frac{1}{2}}(\Gamma)$ anymore, this obviously results in a reduction of the smoothness of $\bar{y}$ since it is not in $H^{1}(\Omega)$. However, for any arbitrary small $\alpha>0$, we have $\bar{u}=-\partial_{\boldsymbol{n}} \bar{p} \in H^{\frac{1}{2}-\alpha}(\Gamma)$ and then $\bar{y} \in H^{1-\alpha}(\Omega)$ (see $\left.[12]\right)$ and $(\bar{y}, \bar{p})$ satisfies the following stability

$$
\|\bar{p}\|_{H^{2}(\Omega)}+\|\bar{y}\|_{H^{1-\alpha}(\Omega)} \leq C(\alpha)\left\|y_{d}\right\|_{L^{2}(\Omega)} .
$$

Things change drastically when $\Omega$ is a non-convex polygon - which may be considered as the generic case -, as the adjoint state is no longer in $H^{2}(\Omega) \cap H_{0}^{1}(\Omega)$. To determine the exact smoothness of $(\bar{y}, \bar{p})$ it is necessary to use the regularizing properties of the Dirichlet-Laplace resolvent for $\bar{p}$ and to resort to additional technical 
manipulations of the coupled problem (5-6). Before stepping further, let us introduce some new notations, the boundary $\Gamma$ is the union of $j^{*}$ segments $\Gamma_{j}\left(1 \leq j \leq j^{*}\right)$ and are numbered in such a way that $\Gamma_{j+1}$ follows $\Gamma_{j}$ according to the positive orientation with the convention that $\Gamma_{j^{*}+1}=\Gamma_{1}$. The corner of the sector described by $\Gamma_{j}$ and $\Gamma_{j+1}$ is denoted $\boldsymbol{c}_{j}$ and the aperture of its angle is $\omega_{j}$. It may occur that at the vicinity of $\boldsymbol{c}_{j}$, we use the polar coordinates so that the set of $(r, \theta)$ with $r \in\left[0, r_{j}\right]$ and $\theta \in\left[0, \omega_{j}\right]$ does not contain any other corner than $\boldsymbol{c}_{j}$. We need also to use an appropriate cut-off function $\chi_{j}$ around $\boldsymbol{c}_{\boldsymbol{j}}$, which is a regular radial dependent function so that $\chi_{j}(r)=1$ for $r \leq \frac{r_{j}}{2}$ and $\chi_{j}(r)=0$ for $r \geq r_{j}$. Finally, we consider three types of singular functions

$$
\begin{aligned}
& S_{j}(r, \theta)=r^{\frac{\pi}{\omega_{j}}} \sin \left(\frac{\pi}{\omega_{j}} \theta\right) \chi_{j}(r), \\
& S_{j}^{*}(r, \theta)=r^{-\frac{\pi}{\omega_{j}}} \sin \left(\frac{\pi}{\omega_{j}} \theta\right) \chi_{j}(r), \\
& T_{j}(r, \theta)=\frac{\pi}{\omega_{j} \sin \left(\frac{\omega_{j}}{2}\right)} r^{\frac{\pi}{\omega_{j}}-1} \sin \left(\left(\frac{\pi}{\omega_{j}}-1\right) \theta+\frac{\omega_{j}}{2}\right) \chi_{j}(r) .
\end{aligned}
$$

We have that $S_{j} \in H^{\gamma}(\Omega)$, for any $\gamma<1+\frac{\pi}{\omega_{j}}, S_{j}^{*} \in H^{\gamma}(\Omega)$, for any $\gamma<1-\frac{\pi}{\omega_{j}}$ and $T_{j} \in H^{\gamma}(\Omega)$, for any $\gamma<\frac{\pi}{\omega_{j}}$.

Changing the functional framework of the saddle point poblem (5-6) to $L^{2}(\Omega) \times\left(H^{2}(\Omega) \cap H_{0}^{1}(\Omega)\right)$ instead of $L^{2}(\Omega) \times D(\Delta)$ produces a new solution $(y, p)(e . g ., p$ is more regular than $\bar{p})$. Comparing it to $(\bar{y}, \bar{p})$ allows to derive the following singular decomposition

$$
\bar{p}=\sum_{j ; \omega_{j}>\pi} \beta_{j} S_{j}+p_{\text {reg }} \quad \text { and } \quad \bar{y}=\sum_{j ; \omega_{j}>\pi}\left(\beta_{j}^{*} S_{j}^{*}+\beta_{j} T_{j}\right)+y_{r e g}
$$

with $p_{\text {reg }} \in H^{2}(\Omega), y_{\text {reg }} \in H^{1-\alpha}(\Omega)$ for any small $\alpha>0$ and the following stability holds

$$
\begin{aligned}
\left\|p_{r e g}\right\|_{H^{2}(\Omega)}+\max _{j ; \omega_{j}>\pi}\left(\left|\beta_{j}\right|\right) & \leq C\left\|y_{d}\right\|_{L^{2}(\Omega)} \\
\left\|y_{r e g}\right\|_{H^{1-\alpha}(\Omega)}+\max _{j ; \omega_{j}>\pi}\left(\left|\beta_{j}^{*}\right|\right) & \leq C(\alpha)\left\|y_{d}\right\|_{L^{2}(\Omega)} .
\end{aligned}
$$

Notice that, around the corner $\boldsymbol{c}_{j}$, the dominant singularity in the expansion of $\bar{y}$ is $S_{j}^{*}$. Defining the regularity exponent $\tau^{*}=\min _{1 \leq j \leq j^{*}}\left(\frac{\pi}{\omega_{j}}\right)<1$, for non-convex domains, it comes out that $\bar{p} \in H^{1+\gamma}(\Omega)$ and $\bar{y} \in H^{\gamma}(\Omega)$ for any $\gamma \in\left[0, \tau^{*}[\right.$.

\section{The PenAlized Robin BOUNDARY CONTROL PROBLEM}

The penalization of the Dirichlet condition by the Robin boundary condition in the state equation may be needed for many good reasons, as evoked in the introduction. The core of this section is to describe the penalization procedure, to recall some proven approximability results and to discuss the consequences induced by the control problem.

When replacing the condition $y_{\mid \Gamma}=u$ by the penalized condition $\left(\varepsilon \partial_{n} y_{\varepsilon}+y_{\varepsilon}\right)_{\mid \Gamma}=u(\varepsilon$ is a positive real number to decay toward zero) in the Poisson problem, we are naturally led to consider the well posed variational problem: find $y_{\varepsilon} \in H^{1}(\Omega)$ such that

$$
\int_{\Omega} \nabla y_{\varepsilon} \nabla z \mathrm{~d} \boldsymbol{x}+\frac{1}{\varepsilon} \int_{\Gamma} y_{\varepsilon} z \mathrm{~d} \Gamma=\frac{1}{\varepsilon} \int_{\Gamma} u z \mathrm{~d} \Gamma, \quad \forall z \in H^{1}(\Omega) .
$$

Before analyzing the control problem, let us define the space $D_{\varepsilon}(\Delta)$, the analogous of $D(\Delta)$ studied in the previous paragraph,

$$
D_{\varepsilon}(\Delta)=\left\{z \in H^{1}(\Omega), \quad \Delta z \in L^{2}(\Omega), \quad\left(\varepsilon \partial_{\boldsymbol{n}} z+z\right)_{\mid \Gamma}=0\right\}
$$


endowed with the norm $\|z\|_{D_{\varepsilon}(\Delta)}:=\|\Delta z\|_{L^{2}(\Omega)}$ which is uniformly (with respect to $\varepsilon$ ) equivalent to the graph norm of $D_{\varepsilon}(\Delta)$ provided that the range of $\varepsilon$ is bounded which is henceforth assumed to be true $\left.(i . e . \varepsilon \in] 0, \varepsilon_{0}\right]$ for a fixed $\left.\varepsilon_{0}\right)$.

Remark 3.1. For regular domains (e.g. of class $\left.\mathscr{C}^{2}\right), D_{\varepsilon}(\Delta)$ coincides with

$$
\left\{z \in H^{2}(\Omega), \quad\left(\varepsilon \partial_{\boldsymbol{n}} z+z\right)_{\mid \Gamma}=0\right\}
$$

and the norm $\|\cdot\|_{D_{\varepsilon}(\Delta)}$ is uniformly equivalent to that induced by $H^{2}(\Omega)$ on $D_{\varepsilon}(\Delta)$ (see $\left.[10]\right)$.

Again, applying Green's formula, the solution $y_{\varepsilon} \in H^{1}(\Omega)$ of $(9)$ also satisfies the $L^{2}$-variational equation:

$$
\int_{\Omega} y_{\varepsilon}(-\Delta z) \mathrm{d} \boldsymbol{x}=-\int_{\Gamma} u\left(\partial_{\boldsymbol{n}} z\right) \mathrm{d} \Gamma, \quad \forall z \in D_{\varepsilon}(\Delta)
$$

The construction of the space $D_{\varepsilon}(\Delta)$ is justified by technical necessity; indeed, it plays an important role for the convergence study of the penalized control toward the non-penalized control we carry out in the next section.

Let us turn back to the penalized optimal control boundary problem that is stated as follows

$$
\begin{gathered}
\min _{u \in L^{2}(\Gamma) ;} J\left(y_{\varepsilon}, u\right) \\
y_{\varepsilon} \text { solution of }(9)
\end{gathered}
$$

Like (1) problem (11) is convex and coercive, and has then only one solution $\bar{u}_{\varepsilon} \in L^{2}(\Gamma)$. The system of optimality conditions can be written down as follows: find $\left(\bar{y}_{\varepsilon}, \bar{p}_{\varepsilon}\right) \in H^{1}(\Omega) \times H^{1}(\Omega)$ such that

$$
\begin{aligned}
\int_{\Omega} \bar{y}_{\varepsilon} z \mathrm{~d} \boldsymbol{x}+\int_{\Omega} \nabla z \nabla \bar{p}_{\varepsilon} \mathrm{d} \boldsymbol{x}+\frac{1}{\varepsilon} \int_{\Gamma} z \bar{p}_{\varepsilon} \mathrm{d} \Gamma & =\int_{\Omega} y_{d} z \mathrm{~d} \boldsymbol{x}, \quad \forall z \in H^{1}(\Omega), \\
\int_{\Omega} \nabla \bar{y}_{\varepsilon} \nabla q \mathrm{~d} \boldsymbol{x}+\frac{1}{\varepsilon} \int_{\Gamma} \bar{y}_{\varepsilon} q \mathrm{~d} \Gamma-\frac{1}{\varepsilon^{2}} \int_{\Gamma} \bar{p}_{\varepsilon} q \mathrm{~d} \Gamma & =0, \quad \forall q \in H^{1}(\Omega),
\end{aligned}
$$

and the optimal control is determined by $\bar{u}_{\varepsilon}=\frac{1}{\varepsilon} \bar{p}_{\varepsilon \mid \Gamma}$. Following the arguments developed for the saddle point problem (5-6), we can prove the Lemma 3.2.

Lemma 3.2. Let $y_{d} \in L^{2}(\Omega)$, problem $(12-13)$ has only one solution $\left(\bar{y}_{\varepsilon}, \bar{p}_{\varepsilon}\right) \in H^{1}(\Omega) \times H^{1}(\Omega)$ such that

$$
\max \left(\left\|\bar{y}_{\varepsilon}\right\|_{L^{2}(\Omega)}, 2\left\|\bar{u}_{\varepsilon}\right\|_{L^{2}(\Gamma)}\right) \leq\left\|y_{d}\right\|_{L^{2}(\Omega)}
$$

Moreover, the adjoint state $\bar{p}_{\varepsilon}$ satisfies

$$
\left\|\bar{p}_{\varepsilon}\right\|_{H^{1}(\Omega)} \leq C\left\|\bar{y}_{d}\right\|_{L^{2}(\Omega)}
$$

the constant $C$ is independent of $\varepsilon$.

By standard manipulations, it can be established that $\bar{p}_{\varepsilon}$ actually belongs to $D_{\varepsilon}(\Delta)$ and that $\left(\bar{y}_{\varepsilon}, \bar{p}_{\varepsilon}\right)$ is the solution of the partial differential system

$$
\begin{aligned}
\bar{y}_{\varepsilon}-\Delta \bar{p}_{\varepsilon} & =y_{d} & & \text { in } \Omega \\
-\Delta \bar{y}_{\varepsilon} & =0 & & \text { in } \Omega \\
\varepsilon \partial_{\boldsymbol{n}} \bar{p}_{\varepsilon}+\bar{p}_{\varepsilon} & =0 & & \text { on } \Gamma \\
\varepsilon \partial_{\boldsymbol{n}} \bar{y}_{\varepsilon}+\bar{y}_{\varepsilon} & =-\partial_{\boldsymbol{n}} \bar{p}_{\varepsilon} & & \text { on } \Gamma,
\end{aligned}
$$


and that $\bar{u}_{\varepsilon}=-\partial_{\boldsymbol{n}} \bar{p}_{\varepsilon}$. The state variable can be eliminated from these equations and we obtain the fourth order problem in the adjoint state: find $\bar{p}_{\varepsilon} \in D_{\varepsilon}(\Delta)$ such that

$$
\int_{\Omega} \Delta \bar{p}_{\varepsilon} \Delta q_{\varepsilon} \mathrm{d} \boldsymbol{x}+\int_{\Gamma}\left(\partial_{\boldsymbol{n}} \bar{p}_{\varepsilon}\right)\left(\partial_{\boldsymbol{n}} q_{\varepsilon}\right) \mathrm{d} \Gamma=\int_{\Omega} y_{d}\left(-\Delta q_{\varepsilon}\right) \mathrm{d} \boldsymbol{x}, \quad \forall q_{\varepsilon} \in D_{\varepsilon}(\Delta) .
$$

Not only does this variational problem produce additional informations about $\bar{p}_{\varepsilon}$,

$$
\left\|\Delta \bar{p}_{\varepsilon}\right\|_{L^{2}(\Omega)} \leq\left\|y_{d}\right\|_{L^{2}(\Omega)}
$$

but it is also adopted in the forthcoming convergence analysis.

\section{Convergence Analysis}

Once the control Dirichlet boundary problem and its Robin penalization are described, we are left with the convergence analysis. Rather than using the mixed problems (5-6) and (12-13) of the optimal conditions, the bilaplacian formulations (7) and (18) are preferred for stating the convergence rate because they are easier to handle. As the results we intend to prove depends on the regularity of the geometry, and so as to avoid high complications and to emphasize on the essential features of the proof, we choose to study the case of smooth domains in details. For polygonal domains, the convergence rate of the penalization is slowed down due to the singularities arisen out at the vicinity of the corner points of the boundary $\Gamma$. We therefore provide only quasi-optimal results.

\subsection{Regular domains}

The assumed domain regularity is that $\Gamma$ is of class $\mathscr{C}^{2}$. We sort among the results of [10] those that are helpful for realizing our project. For any $h \in H^{\frac{1}{2}}(\Gamma)$ the solution of the Poisson-Robin problem

$$
\int_{\Omega} \nabla \tilde{z}_{\varepsilon} \nabla z \mathrm{~d} \boldsymbol{x}+\frac{1}{\varepsilon} \int_{\Gamma} \tilde{z}_{\varepsilon} z \mathrm{~d} \Gamma=\frac{1}{\varepsilon} \int_{\Gamma}(\varepsilon h) z \mathrm{~d} \Gamma, \quad \forall z \in H^{1}(\Omega)
$$

belongs to $H^{2}(\Omega)$ and verifies the estimate (see [10])

$$
\left\|\tilde{z}_{\varepsilon}\right\|_{H^{1+\sigma}(\Omega)} \leq C \varepsilon^{1-\sigma}\|h\|_{H^{\frac{1}{2}}(\Gamma)}
$$

where $\sigma \in[0,1]$ and $C$ does not depend on $\varepsilon$. As a by-product of this, we have the following: let $q_{\varepsilon} \in D_{\varepsilon}(\Delta)$ and $q \in D(\Delta)$ be related to one another by $\left(\Delta q=\Delta q_{\varepsilon}\right)$, then $q$ and $q_{\varepsilon} \in H^{2}(\Omega)$ and the following estimate holds

$$
\left\|q_{\varepsilon}-q\right\|_{H^{1+\sigma}(\Omega)} \leq C \varepsilon^{1-\sigma}\|\Delta q\|_{L^{2}(\Omega)}=C \varepsilon^{1-\sigma}\left\|\Delta q_{\varepsilon}\right\|_{L^{2}(\Omega)} .
$$

Lemma 4.1. Assume that $\Omega$ is of class $\mathscr{C}^{2}$ and that $y_{d} \in L^{2}(\Omega)$. Let $\sigma$ be a given real-number in $[0,1]$, then it holds that

$$
\left\|\bar{p}_{\varepsilon}-\bar{p}\right\|_{H^{1+\sigma}(\Omega)} \leq C \varepsilon^{1-\sigma}\left\|y_{d}\right\|_{L^{2}(\Omega)} .
$$

The constant $C$ is independent of $\varepsilon$.

Proof. For commodity the symbol overline for $\bar{p}$ and $\bar{p}_{\varepsilon}$ is canceled during the proof which is performed in three steps.

Step $i$.- Setting $r_{\varepsilon}=p_{\varepsilon}-p$, then the following Robin condition holds

$$
\varepsilon \partial_{\boldsymbol{n}} r_{\varepsilon}+r_{\varepsilon}=-\varepsilon \partial_{\boldsymbol{n}} p, \quad \text { on } \Gamma
$$


and we have

$$
\int_{\Omega} \Delta r_{\varepsilon} \Delta q_{\varepsilon} \mathrm{d} \boldsymbol{x}+\int_{\Gamma}\left(\partial_{\boldsymbol{n}} r_{\varepsilon}\right)\left(\partial_{\boldsymbol{n}} q_{\varepsilon}\right) \mathrm{d} \Gamma=\int_{\Omega}\left(y_{d}+\Delta p\right)\left(-\Delta q_{\varepsilon}\right) \mathrm{d} \boldsymbol{x}-\int_{\Gamma}\left(\partial_{\boldsymbol{n}} p\right)\left(\partial_{\boldsymbol{n}} q_{\varepsilon}\right) \mathrm{d} \Gamma, \quad \forall q_{\varepsilon} \in D_{\varepsilon}(\Delta) .
$$

Consider $q \in D(\Delta)$ twined to $q_{\varepsilon}$ by the identity $\Delta q=\Delta q_{\varepsilon}$, using the fact that $p$ is the solution of (7) we obtain that

$$
\int_{\Omega} \Delta r_{\varepsilon} \Delta q_{\varepsilon} \mathrm{d} \boldsymbol{x}+\int_{\Gamma}\left(\partial_{\boldsymbol{n}} r_{\varepsilon}\right)\left(\partial_{\boldsymbol{n}} q_{\varepsilon}\right) \mathrm{d} \Gamma=\int_{\Gamma}\left(\partial_{\boldsymbol{n}} p\right)\left(\partial_{\boldsymbol{n}} q-\partial_{\boldsymbol{n}} q_{\varepsilon}\right) \mathrm{d} \Gamma, \quad \forall q_{\varepsilon} \in D_{\varepsilon}(\Delta) .
$$

Step ii .- The non-homogeneous Robin boundary condition (23) is handled using the following artifice: we introduce $\tilde{r}_{\varepsilon}=\left(r_{\varepsilon}-\tilde{z}_{\varepsilon}\right)$, where $\tilde{z}_{\varepsilon}$ is the unique solution to (19) with $h=-\partial_{\boldsymbol{n}} p$. Seen $\partial_{\boldsymbol{n}} p \in H^{\frac{1}{2}}(\Gamma)$ the function $\tilde{z}_{\varepsilon} \in H^{2}(\Omega)$ and on account of $(20)$ we derive that

$$
\left\|\tilde{z}_{\varepsilon}\right\|_{H^{1+\sigma}(\Omega)} \leq C \varepsilon^{1-\sigma}\left\|\partial_{\boldsymbol{n}} p\right\|_{H^{\frac{1}{2}(\Gamma)}} \leq C \varepsilon^{1-\sigma}\|\Delta p\|_{L^{2}(\Omega)} .
$$

This choice makes $\tilde{r}_{\varepsilon}$ be in $D_{\varepsilon}(\Delta)$ and fulfills the variational identity:

$$
\begin{aligned}
\int_{\Omega} \Delta \tilde{r}_{\varepsilon} \Delta q_{\varepsilon} \mathrm{d} \boldsymbol{x}+\int_{\Gamma}\left(\partial_{\boldsymbol{n}} \tilde{r}_{\varepsilon}\right)\left(\partial_{\boldsymbol{n}} q_{\varepsilon}\right) \mathrm{d} \Gamma= & \int_{\Gamma}\left(\partial_{\boldsymbol{n}} p\right)\left(\partial_{\boldsymbol{n}} q-\partial_{\boldsymbol{n}} q_{\varepsilon}\right) \mathrm{d} \Gamma \\
& -\int_{\Gamma}\left(\partial_{\boldsymbol{n}} \tilde{z}_{\varepsilon}\right)\left(\partial_{\boldsymbol{n}} q_{\varepsilon}\right) \mathrm{d} \Gamma, \quad \forall q_{\varepsilon} \in D_{\varepsilon}(\Delta) .
\end{aligned}
$$

Step iii .- Taking $q_{\varepsilon}=\tilde{r}_{\varepsilon}$ in (27) makes sense, setting $q=\tilde{r}$ with $\tilde{r} \in D(\Delta)$ is the function defined by $\Delta \tilde{r}=\Delta \tilde{r}_{\varepsilon}$, and using Cauchy-Schwarz inequality yields

$$
\left\|\Delta \tilde{r}_{\varepsilon}\right\|_{L^{2}(\Omega)}^{2}+\left\|\partial_{\boldsymbol{n}} \tilde{r}_{\varepsilon}\right\|_{L^{2}(\Gamma)}^{2} \leq\left\|\partial_{\boldsymbol{n}} p\right\|_{H^{\frac{1}{2}(\Gamma)}}\left\|\partial_{\boldsymbol{n}}\left(\tilde{r}-\tilde{r}_{\varepsilon}\right)\right\|_{H^{-\frac{1}{2}(\Gamma)}}+\left\|\partial_{\boldsymbol{n}} \tilde{z}_{\varepsilon}\right\|_{H^{-\frac{1}{2}(\Gamma)}}\left\|\partial_{\boldsymbol{n}} \tilde{r}_{\varepsilon}\right\|_{H^{\frac{1}{2}}(\Gamma)} .
$$

Applying the appropriate trace theorems produces the bound

$$
\left\|\Delta \tilde{r}_{\varepsilon}\right\|_{L^{2}(\Omega)}^{2}+\left\|\partial_{\boldsymbol{n}} \tilde{r}_{\varepsilon}\right\|_{L^{2}(\Gamma)}^{2} \leq C\|\Delta p\|_{L^{2}(\Omega)}\left\|\tilde{r}-\tilde{r}_{\varepsilon}\right\|_{H^{1}(\Omega)}+C\left\|\tilde{z}_{\varepsilon}\right\|_{H^{1}(\Omega)}\left\|\Delta \tilde{r}_{\varepsilon}\right\|_{L^{2}(\Omega)} .
$$

Due to estimates (21) and (26) we derive that

$$
\left\|\Delta \tilde{r}_{\varepsilon}\right\|_{L^{2}(\Omega)}^{2}+\left\|\partial_{\boldsymbol{n}} \tilde{r}_{\varepsilon}\right\|_{L^{2}(\Gamma)}^{2} \leq C \varepsilon\|\Delta p\|_{L^{2}(\Omega)}\left\|\Delta \tilde{r}_{\varepsilon}\right\|_{L^{2}(\Omega)},
$$

from which follows the statement

$$
\left\|\Delta \tilde{r}_{\varepsilon}\right\|_{L^{2}(\Omega)} \leq C \varepsilon\|\Delta p\|_{L^{2}(\Omega)} \leq C \varepsilon\left\|y_{d}\right\|_{L^{2}(\Omega)}
$$

The triangular inequality ends to

$$
\left\|r_{\varepsilon}\right\|_{H^{1+\sigma}(\Omega)} \leq\left\|\tilde{r}_{\varepsilon}\right\|_{H^{1+\sigma}(\Omega)}+\left\|\tilde{z}_{\varepsilon}\right\|_{H^{1+\sigma}(\Omega)} \leq C\left(\varepsilon\left\|y_{d}\right\|_{L^{2}(\Omega)}+\varepsilon^{1-\sigma}\left\|y_{d}\right\|_{L^{2}(\Omega)}\right),
$$

which completes the proof.

That the convergence rate is shown on the adjoint state, similar results remain to be established for the optimal control as well as for the optimal state.

Theorem 4.2. Assume that $\Omega$ is of class $\mathscr{C}^{2}$ and that $y_{d} \in L^{2}(\Omega)$. Let $\sigma$ be a given real-number in $[0,1]$, then we have

$$
\left\|\bar{p}_{\varepsilon}-\bar{p}\right\|_{H^{1+\sigma}(\Omega)}+\left\|\bar{u}_{\varepsilon}-\bar{u}\right\|_{H^{-\frac{1}{2}+\sigma}(\Gamma)} \leq C \varepsilon^{1-\sigma}\left\|y_{d}\right\|_{L^{2}(\Omega)}
$$


Furthermore, a similar estimate holds for the optimal state

$$
\left\|\bar{y}_{\varepsilon}-\bar{y}\right\|_{H^{\sigma}(\Omega)} \leq C \varepsilon^{1-\sigma}\left\|y_{d}\right\|_{L^{2}(\Omega)} .
$$

The constant $C$ is independent of $\varepsilon$.

Proof. From the essential observation $\bar{u}_{\varepsilon}-\bar{u}=-\left(\partial_{\boldsymbol{n}} p_{\varepsilon}-\partial_{\boldsymbol{n}} p\right)=-\partial_{\boldsymbol{n}} r_{\varepsilon}$, we can write that

$$
\left\|\partial_{\boldsymbol{n}} r_{\varepsilon}\right\|_{H^{-\frac{1}{2}+\sigma}(\Gamma)} \leq\left\|\partial_{\boldsymbol{n}} \tilde{z}_{\varepsilon}\right\|_{H^{-\frac{1}{2}+\sigma}(\Gamma)}+\left\|\partial_{\boldsymbol{n}} \tilde{r}_{\varepsilon}\right\|_{H^{-\frac{1}{2}+\sigma}(\Gamma)} .
$$

Invoking the continuity of the normal derivative operator, we obtain

$$
\left\|\partial_{\boldsymbol{n}} r_{\varepsilon}\right\|_{H^{-\frac{1}{2}+\sigma}(\Gamma)} \leq C\left(\left\|\tilde{z}_{\varepsilon}\right\|_{H^{1+\sigma}(\Omega)}+\left\|\Delta \tilde{r}_{\varepsilon}\right\|_{L^{2}(\Omega)}\right) .
$$

Owing to (26) and to (28), the estimate on the control is completed. In the other side observing that $\left(\bar{y}_{\varepsilon}-\bar{y}\right)$ is the solution to :

$$
\begin{aligned}
-\Delta\left(\bar{y}_{\varepsilon}-\bar{y}\right) & =0, \quad \text { in } \Omega \\
\varepsilon \partial_{\boldsymbol{n}}\left(\bar{y}_{\varepsilon}-\bar{y}\right)+\left(\bar{y}_{\varepsilon}-\bar{y}\right) & =\left(\bar{u}_{\varepsilon}-\bar{u}\right)-\varepsilon \partial_{\boldsymbol{n}} \bar{y}, \quad \text { on } \Gamma,
\end{aligned}
$$

it follows that, using [10], that

$$
\left\|\bar{y}_{\varepsilon}-\bar{y}\right\|_{H^{\sigma}(\Omega)} \leq C \varepsilon^{-\sigma}\left(\left\|\bar{u}_{\varepsilon}-\bar{u}\right\|_{H^{-\frac{1}{2}(\Gamma)}}+\varepsilon\left\|\partial_{\boldsymbol{n}} \bar{y}\right\|_{H^{-\frac{1}{2}(\Gamma)}}\right) .
$$

Due to (29), we obtain an estimate on the optimal solution and the proof is complete.

Remark 4.3. For the limit value of $\sigma=1$, the theorem says that $\left(\bar{p}_{\varepsilon}, \bar{u}_{\varepsilon}, \bar{y}_{\varepsilon}\right)$ converges weakly toward $(\bar{p}, \bar{u}, \bar{y})$ in $H^{2}(\Omega) \times H^{\frac{1}{2}}(\Gamma) \times H^{1}(\Omega)$. Actually, it may be proven, by a density argument, that the strong convergence holds too.

Sometimes the domain $\Omega$ and the profile $y_{d}$ are more regular, then higher asymptotic expansions with respect to $\varepsilon$ can possibly be investigated using the power series; it is worth highlighting the final results that are specific to this situation.

Assume then that $\Omega$ is of class $\mathscr{C}^{m+2}$ and that $y_{d} \in H^{m}(\Omega)$ for a given integer $m$. Obvious induction proves that $\left(\bar{p}_{\varepsilon}, \bar{u}_{\varepsilon}, \bar{y}_{\varepsilon}\right)$ belongs to $H^{m+2}(\Omega) \times H^{m+\frac{1}{2}}(\Gamma) \times H^{m+1}(\Omega)$. This suggests that $\left(\bar{p}_{\varepsilon}, \bar{u}_{\varepsilon}, \bar{y}_{\varepsilon}\right)$ be decomposed as

$$
\begin{aligned}
& \bar{p}_{\varepsilon}=\bar{p}+\varepsilon \bar{p}_{1}+\cdots+\varepsilon^{m} \bar{p}_{m}+\bar{r}_{\varepsilon}^{p} \\
& \bar{y}_{\varepsilon}=\bar{y}+\varepsilon \bar{y}_{1}+\cdots+\varepsilon^{m} \bar{y}_{m}+\bar{r}_{\varepsilon}^{y} \\
& \bar{u}_{\varepsilon}=\bar{u}+\varepsilon \bar{u}_{1}+\cdots+\varepsilon^{m} \bar{u}_{m}+\bar{r}_{\varepsilon}^{u}
\end{aligned}
$$

where the residuals $\left(\bar{r}_{\varepsilon}^{p}, \bar{r}_{\varepsilon}^{y}, \bar{r}_{\varepsilon}^{u}\right)$ are assumed to be $o\left(\varepsilon^{m}\right)$. Of course, the first term of the expansion coincides with the solution $(\bar{p}, \bar{u}, \bar{y})$ of the non-penalized control problem. Inserting this expansion in (14-17) and identifying the powers of $\varepsilon$ we obtain the following characterization of the states $\left(\bar{p}_{k}, \bar{y}_{k}\right)$ and the control $\bar{u}_{k}$ of the expansion: for any $k(1 \leq k \leq m)$

$$
\begin{array}{rlrl}
\bar{y}_{k}-\Delta \bar{p}_{k} & =0 \quad \text { in } \Omega & \\
-\Delta \bar{y}_{k} & =0 \quad \text { in } \Omega & \\
\bar{p}_{k} & =-\partial_{\boldsymbol{n}} \bar{p}_{k-1} \quad & & \text { on } \Gamma \\
\bar{y}_{k}+\partial_{\boldsymbol{n}} \bar{p}_{k} & =-\partial_{\boldsymbol{n}} \bar{y}_{k-1} & & \text { on } \Gamma,
\end{array}
$$


with $\bar{u}_{k}=-\partial_{n} \bar{p}_{k}$ where upon the residuals $\left(\bar{r}_{\varepsilon}^{p}, \bar{r}_{\varepsilon}^{y}\right)$ are solutions of

$$
\begin{array}{rlrl}
\bar{r}_{\varepsilon}^{y}-\Delta \bar{r}_{\varepsilon}^{p} & =0 \quad \text { in } \Omega & \\
-\Delta \bar{r}_{\varepsilon}^{y} & =0 \quad \text { in } \Omega & \\
\varepsilon \partial_{\boldsymbol{n}} \bar{r}_{\varepsilon}^{p}+\bar{r}_{\varepsilon}^{p} & =-\varepsilon^{m+1} \partial_{\boldsymbol{n}} \bar{p}_{m} & & \text { on } \Gamma \\
\varepsilon \partial_{\boldsymbol{n}} \bar{r}_{\varepsilon}^{y}+\bar{r}_{\varepsilon}^{y} & =-\partial_{\boldsymbol{n}} \bar{r}_{\varepsilon}^{p}-\varepsilon^{m+1} \partial_{\boldsymbol{n}} \bar{y}_{m} & & \text { on } \Gamma,
\end{array}
$$

with $\bar{r}_{\varepsilon}^{u}=-\partial_{\boldsymbol{n}} \bar{r}_{\varepsilon}^{p}$.

By the elliptic regularity together with an induction argument, we have $\left(\bar{p}_{k}, \bar{y}_{k}\right) \in H^{m+2-k}(\Omega) \times H^{m+1-k}(\Omega)$ and consequently $\bar{u}_{k} \in H^{m+\frac{1}{2}-k}(\Gamma)$. Similarly, we can establish that $\left(\bar{r}_{\varepsilon}^{p}, \bar{r}_{\varepsilon}^{y}, \bar{r}_{\varepsilon}^{u}\right) \in H^{2}(\Omega) \times H^{1}(\Omega) \times H^{\frac{1}{2}}(\Gamma)$. Additionally, as previously seen, an equivalent formulation of this system is obtained by eliminating $\bar{r}_{\varepsilon}^{y}$, which leads to the fourth order elliptic variational equation: find $\bar{r}_{\varepsilon}^{p} \in H^{2}(\Omega) ; \varepsilon \partial_{\boldsymbol{n}} \bar{r}_{\varepsilon}^{p}+\bar{r}_{\varepsilon}^{p}=-\varepsilon^{m+1} \partial_{\boldsymbol{n}} \bar{p}_{m}$ such that

$$
\int_{\Omega} \Delta \bar{r}_{\varepsilon}^{p} \Delta q_{\varepsilon} \mathrm{d} \boldsymbol{x}+\int_{\Gamma}\left(\partial_{\boldsymbol{n}} \bar{r}_{\varepsilon}^{p}\right)\left(\partial_{\boldsymbol{n}} q_{\varepsilon}\right) \mathrm{d} \Gamma=-\int_{\Gamma}\left(\varepsilon^{m+1} \partial_{\boldsymbol{n}} \bar{y}_{m}\right)\left(\partial_{\boldsymbol{n}} q_{\varepsilon}\right) \mathrm{d} \Gamma, \quad \forall q_{\varepsilon} \in D_{\varepsilon}(\Delta) .
$$

Following the lines of the proof of Lemma 4.1 and Theorem 4.2, it can be proven that

Theorem 4.4. Assume that $\Omega$ is of class $\mathscr{C}^{m+2}$ and that $y_{d} \in H^{m}(\Omega)$ and let $\sigma$ be a real-number given in $[0,1]$. Then the asymptotic expansion holds true and we have the following error estimate

$$
\left\|\bar{r}_{\varepsilon}^{p}\right\|_{H^{1+\sigma}(\Omega)}+\left\|\bar{r}_{\varepsilon}^{y}\right\|_{H^{\sigma}(\Omega)}+\left\|\bar{r}_{\varepsilon}^{u}\right\|_{H^{-\frac{1}{2}+\sigma}(\Gamma)} \leq C \varepsilon^{m+1-\sigma}\left\|y_{d}\right\|_{H^{m}(\Omega)}
$$

The constant $C$ is independent of $\varepsilon$.

Remark 4.5. The results obtained in this section for regular two-dimensional domains are valid as well for the three-dimensional case provided that the same geometrical regularity assumptions are available.

Remark 4.6. The Robin penalization was used in the optimal boundary control flows $([13,14])$ and gave satisfactory results. The analysis detailed for the Poisson problem is readily extended to the linear Stokes system. For regular domains, this penalization procedure yields also a linear convergence with respect to the penalization parameter $\varepsilon$.

\subsection{Some words about the polygonal domains}

Lemma 4.1 and Theorem 4.2, where the asymptotics of the penalized solution of the control problem are given, are specific to the regular domains. Stating high order expansion for non-smooth domains, in particular for polygons, is involved with the singular functions and requires high technical analysis similar to that made in [10]; this is beyond the aim of this work and is not undertaken. However, in continuation of Section 2.2, we provide sub-optimal convergence results, for the first order expansion (8), which can be obtained following the proof detailed in the regular case, taking into account the loss of regularity caused by the non-smoothness of the domain (see [12]).

In view of the discussion of Section 2.2 , the regularity $(\bar{y}, \bar{p}) \in H^{1}(\Omega) \times H^{2}(\Omega)$ is not valid as soon as $\Omega$ is a polygon. Therefore, estimate (22) fails and should be modified, because the function $\partial_{\boldsymbol{n}} \bar{p}$ cannot belong to $H^{\frac{1}{2}}(\Gamma)$ any longer. Actually, we have $\partial_{\boldsymbol{n}} \bar{p} \in H^{-\frac{1}{2}+\tau^{*}-\alpha}(\Gamma)$ for any small $\alpha>0$ with $\tau^{*}=\min _{1 \leq j \leq j^{*}}\left(\frac{\pi}{\omega_{j}}\right)$, even though for convex sets the inclusion $\left(\partial_{\boldsymbol{n}} \bar{p}\right)_{\mid \Gamma_{j}} \in H^{\frac{1}{2}}\left(\Gamma_{j}\right)$ holds locally. This has a direct impact on estimate (26) which is transformed into

$$
\left\|\tilde{z}_{\varepsilon}\right\|_{H^{1+\sigma}(\Omega)} \leq C(\alpha) \varepsilon^{-\alpha} \varepsilon^{\tau^{*}-\sigma}\|\Delta \bar{p}\|_{L^{2}(\Omega)}
$$

for arbitrary small $\alpha>0$. Working with (33) instead of (26) gives the result of the following theorem 
Theorem 4.7. Assume that $\Omega$ is a Lipschitz polygonal domain and $y_{d} \in L^{2}(\Omega)$. Let $\sigma$ be a given real-number in $\left[0, \tau^{*}[\right.$, then we have

$$
\left\|\bar{p}_{\varepsilon}-\bar{p}\right\|_{H^{1+\sigma}(\Omega)}+\left\|\bar{u}_{\varepsilon}-\bar{u}\right\|_{H^{-\frac{1}{2}+\sigma}(\Gamma)}+\left\|\bar{y}_{\varepsilon}-\bar{y}\right\|_{H^{\sigma}(\Omega)} \leq C(\alpha) \varepsilon^{-\alpha} \varepsilon^{\tau^{*}-\sigma}\left\|y_{d}\right\|_{L^{2}(\Omega)},
$$

for $\alpha$ arbitrary small positive real-number and the constant $C$ depends on $\alpha$.

Proof. The proof is structured as in the regular case. We first state the result on the adjoint state following the lines of the proof of Lemma 4.1 with the necessary adaptations. The estimates on the control $\bar{u}$ and the direct state $\bar{y}$ stem from the estimate on $\bar{p}$.

Remark 4.8. The constant $C(\alpha)$ is supposed to blow-up when $\alpha$ decays to zero. Estimates (33) and (34) do not seem optimal and we conjecture that by working out the technical details as done in [10] it would be possible to improve the convergence rate, getting rid of the extra-term $\varepsilon^{-\alpha}$ which could be replaced by a (poly-) logarithmic term. Then, estimate (34) could be stated to

$$
\left\|\bar{p}_{\varepsilon}-\bar{p}\right\|_{H^{1+\sigma}(\Omega)}+\left\|\bar{u}_{\varepsilon}-\bar{u}\right\|_{H^{-\frac{1}{2}+\sigma}(\Gamma)}+\left\|\bar{y}_{\varepsilon}-\bar{y}\right\|_{H^{\sigma}(\Omega)} \leq C \varepsilon^{\tau^{*}-\sigma}|\log \varepsilon|^{\gamma}\left\|y_{d}\right\|_{L^{2}(\Omega)},
$$

$\gamma$ is a positive exponent.

\section{NumericAl INVESTIGATION}

In the numerical investigation, we pursue two goals. We start by two experiments on the boundary control problem, one is related to a very smooth solution and for the other the solution contains a singular part. We compare the computed convergence rates with the theoretical results predicted in Theorem 4.2 and Theorem 4.7. Next, to emphasize the reliability of our penalization procedure we figure out how it performs in some special cases for which the results of Section 4 fail to predict how things happen.

Let $\Omega$ be the $L$-shaped domain $[0,1]^{2} \backslash\left[0, \frac{1}{2}\right]^{2}$ and we begin by the example where the optimal state and the optimal control to be simulated are

$$
\bar{z}(x, y)=\cos (x) \sin (y) \quad \text { and } \quad \bar{u}(x, y)=(\bar{z}(x, y))_{\mid \Gamma},
$$

which are very smooth. Then, we set $z_{d}=\bar{z}, u_{d}=\bar{u}$ and we consider the following optimal control problem

$$
\begin{gathered}
\min \quad \frac{1}{2} \int_{\Omega}\left(z-z_{d}\right)^{2} \mathrm{~d} \boldsymbol{x}+\frac{\beta}{2} \int_{\Gamma}\left(u-u_{d}\right)^{2} \mathrm{~d} \Gamma \\
\left\{\begin{array}{cl}
-\Delta z=2 z_{d}, & \text { in } \Omega \\
z=u, & \text { on } \Gamma .
\end{array}\right.
\end{gathered}
$$

It is easily checked that the minimum value of the objective function is zero and that it is reached at $\left(z_{d}, u_{d}\right)$. The calculations are made by a standard Galerkin linear finite elements. The triangular mesh is constructed on a uniform quadrangular mesh ( $c f$. Fig. 1), where each quadrangle is split into two triangles, and the mesh size is $h=0.0125$. The minimum of the objective function is computed by a conjugate gradient method where, at each iteration, the discrete algebraic Robin-Poisson problem is solved using the Choleski factorization - which is accomplished in the pre-processing step - since the associated matrix is symmetric and positive definite.

We take $\beta=1$, and for different values of the penalization parameter $\varepsilon$, we compute the discrete optimal state $\bar{z}_{\varepsilon}^{h}$ and the discrete optimal control $\bar{u}_{\varepsilon}^{h}$. In Figure 2, are plotted, in a logarithmic scale, the behavior of the relative error functions

$$
\varepsilon \mapsto \frac{\left\|\bar{z}-\bar{z}_{\varepsilon}^{h}\right\|}{\|\bar{z}\|}, \quad \varepsilon \mapsto \frac{\left\|\bar{u}-\bar{u}_{\varepsilon}^{h}\right\|}{\|\bar{u}\|}
$$



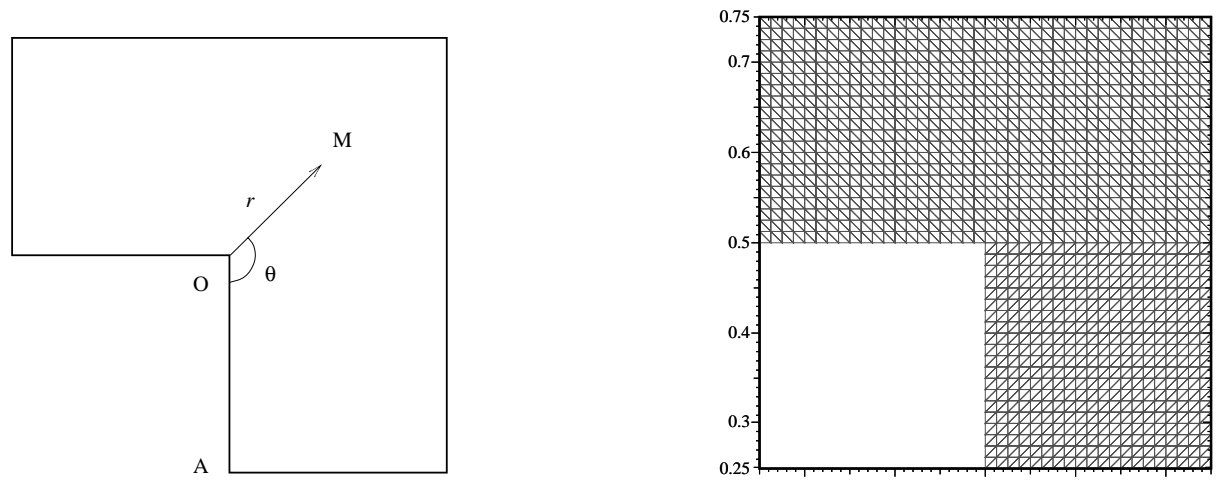

FiguRE 1. The $L$-shaped domain and a zoom on the mesh.

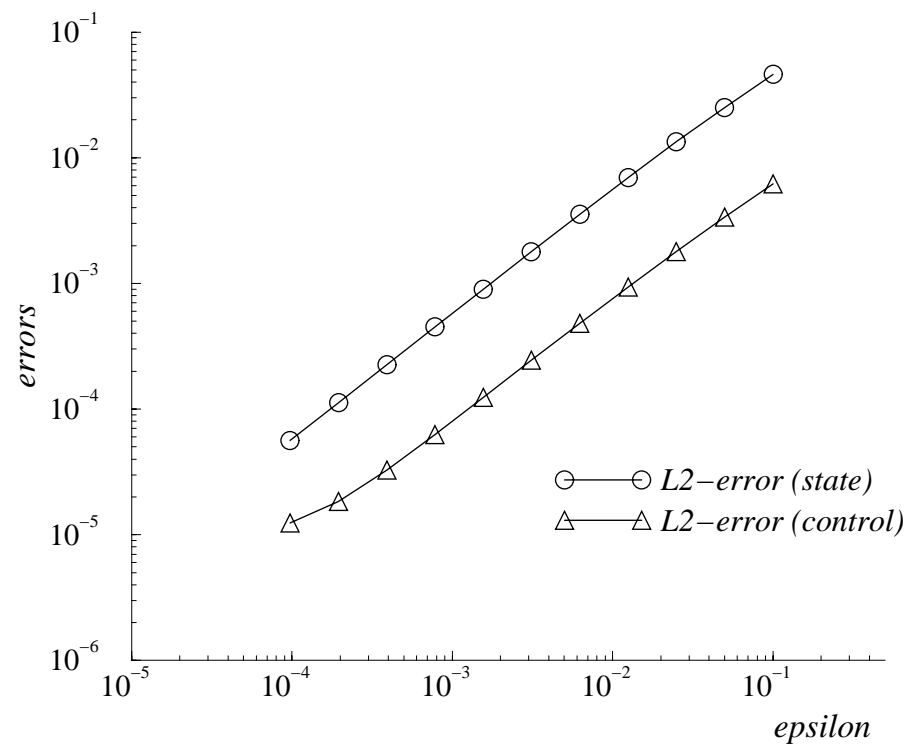

Figure 2. Convergence curves for a smooth solution. The slopes indicate a linear convergence of the penalization on the optimal control and the optimal state.

in the $L^{2}$-norm for the optimal state and in the $L^{2}$-norm for the optimal control. From Theorem 4.2 , these errors are expected to decay linearly with respect to $\varepsilon$, this fact can be observed in the figure. Furthermore, we have measured the slopes of the linear regression of the two curves - they are also the convergence rates and we found that both of them are very close to one, which attest for a linear convergence. Notice that the error curve on the optimal control is beginning to flatten out near the value $\varepsilon=10^{-4}$. The explanation is that, at this level, there is a balance between the finite element error and the penalization error; for lower values of the penalization parameter $\varepsilon<10^{-4}$ the errors on both optimal control and optimal state reach a plateau corresponding the finite element contribution to the global error.

In the second example, we deal with a non-smooth solution set on the same $L$-shaped domain $\Omega$, that is generated by the non-convex corner $O\left(\frac{1}{2}, \frac{1}{2}\right)$. We consider the case where the profile is the singular harmonic function

$$
z_{d}(x, y)=r^{\frac{2}{3}} \sin \left(\frac{2}{3} \theta\right) \quad \text { and } \quad u_{d}(x, y)=\left(z_{d}(x, y)\right)_{\mid \Gamma} .
$$




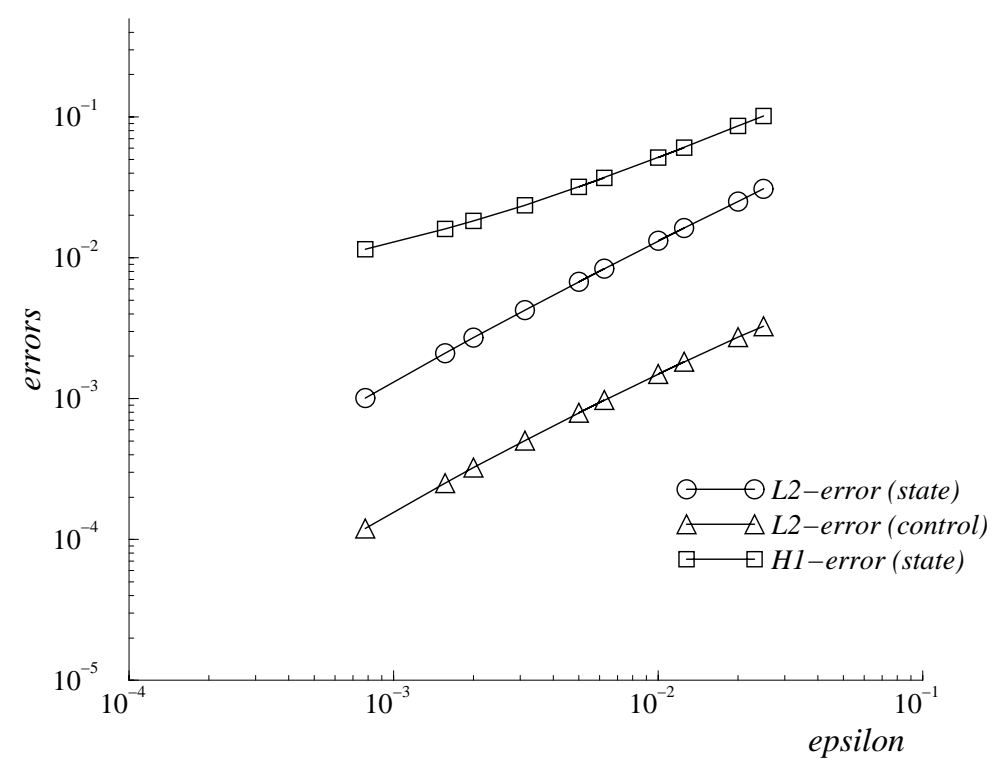

Figure 3. Convergence curves for a non-smooth solution. The $H^{1}$-error on the optimal state is affected by the corner singularity.

$(r, \theta)$ are the polar coordinates of $M(x, y), r$ is the distance between $M$ and $O$ and $\theta$ is the measure of the aperture of the angle between the lines $(O A)$ and $(O M)$ where $A\left(\frac{1}{2}, 0\right)$. The solution of the non-penalized optimal control problem, with the same objective function, is given by $(\bar{z}, \bar{u})=\left(z_{d}, u_{d}\right)$ - note that, here, the adjoint state has the same regularity as $\bar{z}=z_{d}$. We intend to compare the penalized solution to the exact one. To obtain sufficient accuracy of the finite element approximation, the computations are carried out using a triangular mesh of size twice smaller than that of the first example $(h=0.00625)$, because of the lack of regularity of the exact solution. In Figure 3, the $L^{2}$-norm and the $H^{1}$-norm of the error between $\bar{z}$ and $\bar{z}_{\varepsilon}^{h}$ are depicted, which are expected to decay like $\varepsilon$ and $\varepsilon^{2 / 3}$ respectively, and the $L^{2}$-norm of the error on the optimal control $\left(\bar{u}-\bar{u}_{\varepsilon}^{h}\right)$ which shall decrease toward zero like $\varepsilon$. The evaluation of the slopes of the linear regression corresponding to the three graphs are in accordance with the predictions, the slopes are given by $0.65(\approx 0.66)$ and $0.97(\approx 1)$ for the $H^{1}$-norm and for the $L^{2}$-norm of the error on the optimal state respectively, and for the optimal control the slope is $0.95(\approx 1)$.

Actually, since in the most general situations the exact solution is out of access, then we cannot compare the computed control $\bar{u}_{\varepsilon}^{h}$ to the exact control $\bar{u}$. The most informative question becomes: how close is the penalized finite element solution $\left(\bar{u}_{\varepsilon}^{h}, \bar{z}_{\varepsilon}^{h}\right)$ to the non-penalized finite element solution $\left(\bar{u}^{h}, \bar{z}^{h}\right.$ ) (for which the exact Dirichlet condition is enforced)?

In Figure 4, the difference between both solutions in different norms are plotted with respect to the parameter $\varepsilon$, in logarithmic scales. At the first glance, the different errors seem to decay towards zero with the same rate until the formation of a plateau at a low level $\left(\approx 10^{-8}\right)$. This trend is confirmed by the computation of the slopes of all the curves far from the plateau, we obtain 0.98 for the $H^{1}$-norm on $\bar{z}^{h}, 0.99$ for both the $L^{2}$-norm on $\bar{z}^{h}$ and the $L^{2}$-norm on $\bar{u}^{h}$. Contrary to the comparison with the exact solution, the convergence here is linear in $\varepsilon$ for different norms, even for $H^{1}$-norm. Recalling that one of the major practical motivations of the penalization is that it is easier to code a Robin condition - versus the Dirichlet condition - in a computing code, these observations are very encouraging for choosing this approach.

We end the numerical section by discussing the example worked out in [5]. The goal is to highlight the efficiency of the penalization procedure for a non-linear optimal control problem. In the context we are interested in, the non-linearity is due to the inequality constraints on both the state and the control variables. 


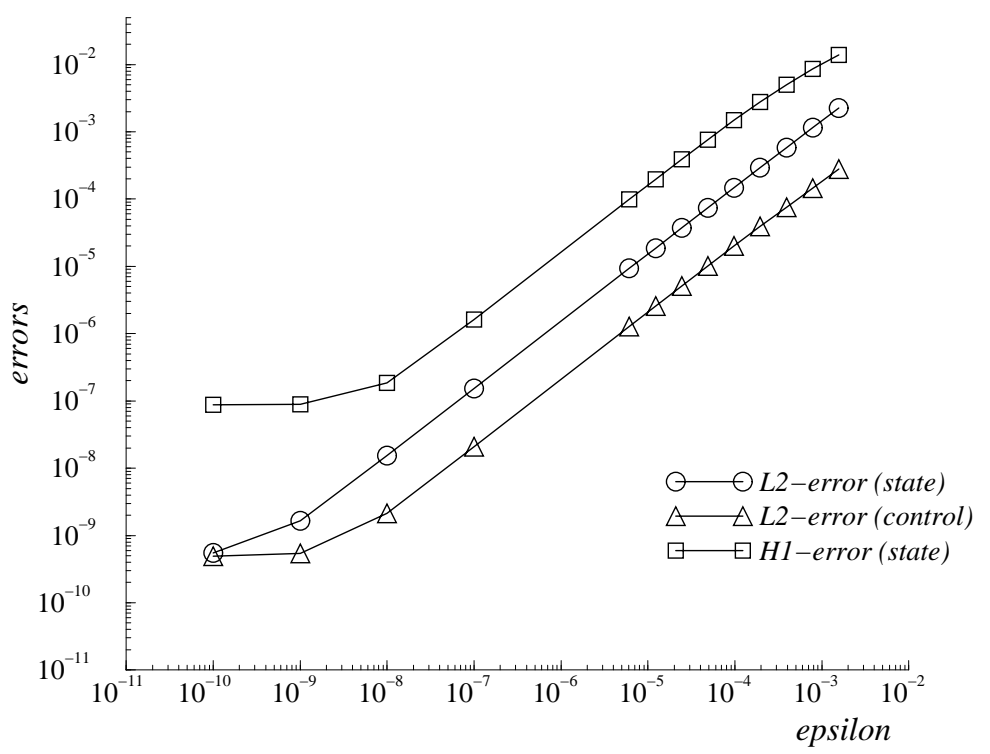

FigurE 4. The gap between the computed penalized and non-penalized solutions (the same solution as in Fig. 3). A linear convergence rate is observed despite the singularity occurring.

Let $\Omega$ be the square $[0,1]^{2}$, we consider the problem of minimizing the objective function of $(35)$ with $u_{d}=0$, where $(z, u)$ are such that

$$
\begin{array}{rlrl}
-\Delta z & =20 & & \text { in } \Omega \\
z & =u & & \text { on } \Gamma \\
z_{\min } \leq z \leq z_{\max } & & \text { in } \Omega \\
u_{\min } \leq u \leq u_{\max } & & \text { on } \Gamma .
\end{array}
$$

An equivalent formulation is obtained by eliminating the state $z$ by using the resolvent of the Dirichlet-Poisson equation (36-37), (i.e. $z=\mathcal{R}(u)$ ). The control problem can, then, be written down only in term of $u$ : find $\bar{u} \in L^{2}(\Gamma)$ verifying

$$
\begin{gathered}
\text { min } \\
\left\{\begin{array}{cl}
z_{\min } \leq \mathcal{R}(u) \leq z_{\max }, & \text { in } \Omega \\
u_{\min } \leq u \leq u_{\max }, & \text { on } \Gamma .
\end{array}\right.
\end{gathered}
$$

The penalized problem can be put under the same form, apart the fact that the resolvent $\mathcal{R}_{\varepsilon}$ involved in it is related to the Robin-Poisson problem, where the Dirichlet condition (37) is changed to $\left(\varepsilon \partial_{\boldsymbol{n}} z_{\varepsilon}+z_{\varepsilon}\right)_{\mid \Gamma}=u$. The methodology followed to underline the performances of our approach is to compute the non-penalized optimal control together with the penalized control and their corresponding optimal states, and to look at the variation of the gap between them with respect to the parameter $\varepsilon$. For a given datum $u$, the solutions $z^{h}=\mathcal{R}^{h}(u)$ and $z_{\varepsilon}^{h}=\mathcal{R}_{\varepsilon}^{h}(u)$ are computed by the linear finite element method on a uniform triangular mesh with size $h=0.0125$. The evaluation of the minimum of the objective function, in both cases (the penalized and the non-penalized) is made by the sequential quadratic programming (SQP) algorithm from the NAG library. This requires to provide, in addition to the finite element solvers $\mathcal{R}^{h}$ and $\mathcal{R}_{\varepsilon}^{h}$, the calculation of the adjoint states of the constraints (38) which are incorporated in our Fortran program. For the computations we keep the data 


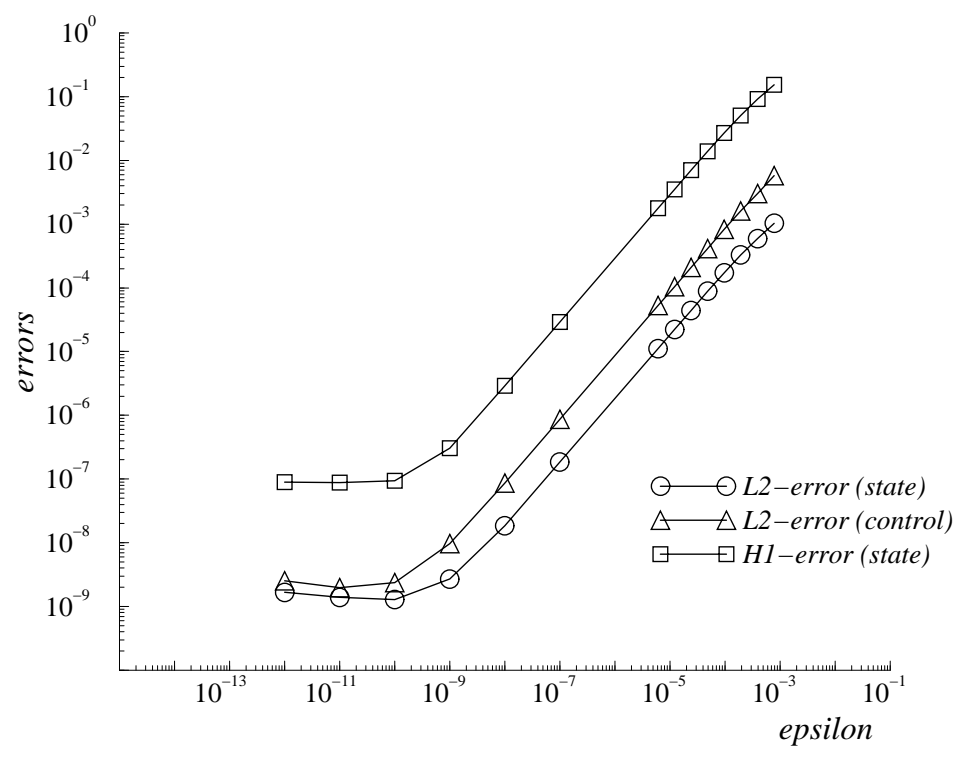

Figure 5. A linear decreasing of the penalization error is achieved for the non-linear constrained boundary control problem.

experimented in [5], $\beta$ is fixed to 0.01 and

$$
z_{d}(x, y)=5 x(x-1) y(y-1)+3, \quad\left(u_{\min }, u_{\max }\right)=(-10,10), \quad\left(z_{\min }, z_{\max }\right)=(0,3.5) .
$$

In Figure 5, the errors between the non-penalized solution (the Dirichlet datum is enforced exactly) and the penalized solution are represented in logarithmic scales. The evaluation of the linear regression of the different curves yields the following conclusions, the slopes for the $H^{1}$-norm on $\bar{z}^{h}$, the $L^{2}$-norm on $\bar{z}^{h}$ and $L^{2}$-norm on $\bar{u}^{h}$ are respectively $0.98,0.99$ and 0.97 . This allows to conjecture that in this example, the penalized (Robin) solution converges towards the (Dirichlet) solution like $\varepsilon$.

\section{CONCLUding REMARKS AND SOME EXTENSIONS}

The Robin penalization technique is a popular process for different type of partial differential equations in order to take into account Dirichlet boundary conditions, in particular for non-smooth data. In this contribution, we rigorously prove the reliability of this approach for the boundary control problem by stating the expected asymptotic convergence rate of the penalized solution toward the exact solution. These predictions are attested by various computational experiments. Furthermore, when applied to a non-linear example, the numerical results confirm the efficiency of the penalization, even though theoretical predictions are missing for this case.

The penalization of the boundary conditions can be extended to different contexts in the optimal control field, such as the distributed control problem. For conciseness, we restrict ourselves to the indications that seem important to establish optimal convergence rates in this case. The objective function is changed to

$$
J(z, \psi)=\frac{1}{2} \int_{\Omega}\left(z-z_{d}\right)^{2} \mathrm{~d} \boldsymbol{x}+\frac{\beta}{2} \int_{\Omega}\left(\psi-\psi_{d}\right)^{2} \mathrm{~d} \boldsymbol{x}
$$

and the partial differential equation reads as

$$
\begin{aligned}
-\Delta z=\psi & \text { in } \Omega, \\
z=0 & \text { on } \Gamma,
\end{aligned}
$$




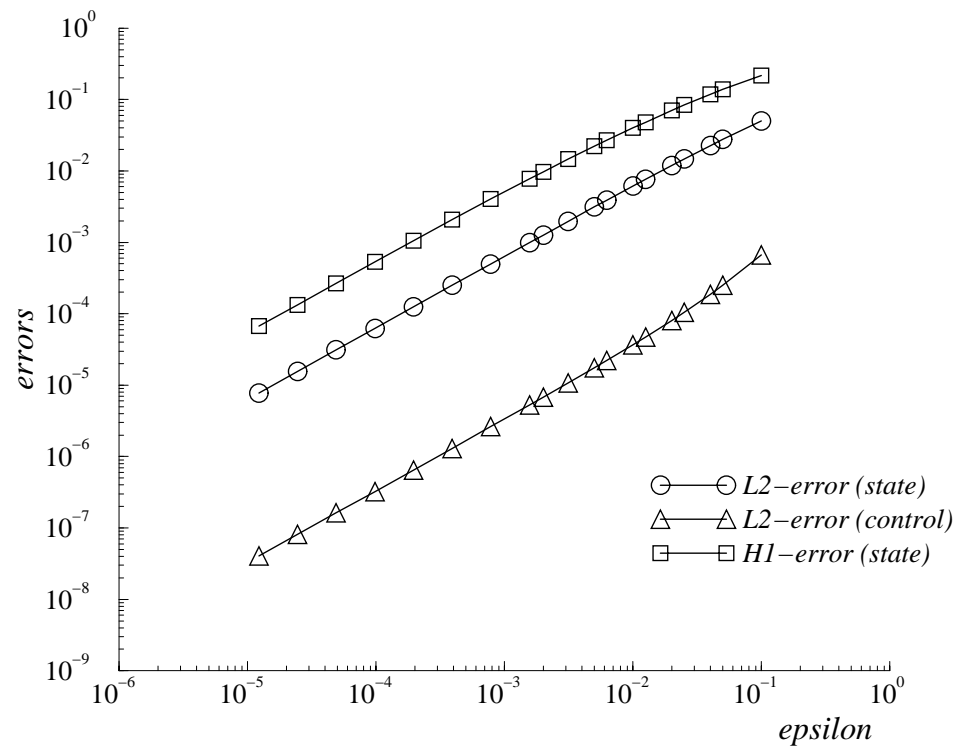

Figure 6. Convergence curve in the case of a distributed control. A linear decreasing of the penalization error is also observed in this case.

where $\psi \in L^{2}(\Omega)$ is the distributed control. Introducing the penalization term transforms the Dirichlet condition into the Robin condition $\left(\varepsilon \partial_{\boldsymbol{n}} z_{\varepsilon}+z_{\varepsilon}\right)_{\mid \Gamma}=0$. Writing down the optimality conditions and eliminating $z$ and $\psi$ provide the following fourth order variational problem on the adjoint state: find $\bar{p} \in D(\Delta)$ such that

$$
\int_{\Omega} \Delta \bar{p} \Delta q \mathrm{~d} \boldsymbol{x}+\frac{1}{\beta} \int_{\Omega} \bar{p} q \mathrm{~d} \boldsymbol{x}=\int_{\Omega} z_{d}(-\Delta q) \mathrm{d} \boldsymbol{x}-\frac{1}{\beta} \int_{\Omega} \psi_{d} q \mathrm{~d} \boldsymbol{x}, \quad \forall q \in D(\Delta)
$$

The penalized adjoint state $\bar{p}_{\varepsilon}$ solves the same variational problem set on $D_{\varepsilon}(\Delta)$ instead of $D(\Delta)$. The optimal controls are obtained thanks to the formulas $\bar{\psi}=\psi_{d}+\frac{1}{\beta} \bar{p}$ and $\bar{\psi}_{\varepsilon}=\psi_{d}+\frac{1}{\beta} \bar{p}_{\varepsilon}$. The convergence rates induced by the penalization can be exhibited following the analysis developed in Section 3. We obtain the desired estimates on the control variables and on the optimal states

$$
\left\|\bar{\psi}_{\varepsilon}-\bar{\psi}\right\|_{L^{2}(\Omega)}+\left\|\bar{z}_{\varepsilon}-\bar{z}\right\|_{H^{1}(\Omega)} \leq C \varepsilon\left(\left\|z_{d}\right\|_{L^{2}(\Omega)}+\left\|\psi_{d}\right\|_{L^{2}(\Omega)}\right)
$$

A numerical experiment is made to certify this estimate. The domain $\Omega$ is the square $] 0,1\left[^{2}\right.$ and the data are

$$
z_{d}(x, y)=\cos (x) \sin (y) \quad \text { and } \quad \psi_{d}(x, y)=2 z_{d}(x, y)
$$

and the exact solution is given by $(\bar{z}, \bar{\psi})=\left(z_{d}, \psi_{d}\right)$. In Figure 6 we compare the penalized computed solution with the Dirichlet computed one. As expected, the errors decay toward zero linearly, with respect to different norms.

The next step to be worked out is to look at the extension of the Robin-penalization procedure to the boundary optimal control of non-stationary equations such as the heat equation, the Stokes and the NavierStokes systems. Some numerical tests are already realized on the heat equation in order to figure out the convergence rate of the penalization, and yield the expected results. The proof of its convergence in a weak sense is established in [2] and stating a linear convergence rate with respect to the penalization parameter is under consideration in our team. 
Acknowledgements. The LAMSIN's researcher's work is supported by the Secrétariat d'état de la recherche scientifique et de la technologie (SERST), within the LAB-STI-02 program. This work was partially supported by the Comité Mixte Franco-Tunisien pour la Coopération Universitaire under CMCU 02F1503 project.

\section{REFERENCES}

[1] D.A. Adams, Sobolev Spaces. Academic Press, New York (1975).

[2] N. Arada, H. El Fekih and J.-P. Raymond, Asymptotic analysis of some control problems. Asymptot. Anal. 24 (2000) $343-366$.

[3] I. Babuška, The finite element method with penalty. Math. Comp. 27 (1973) 221-228.

[4] F. Ben Belgacem, H. El Fekih and J.-P. Raymond, A penalized Robin approach for solving a parabolic equation with nonsmooth Dirichlet boundary conditions. Asymptot. Anal. 34 (2003) 121-136.

[5] M. Bergounioux and K. Kunisch, Augmented Lagrangian techniques for elliptic state constrained optimal control problems. SIAM J. Control Optim. 35 (1997) 1524-1543.

[6] A. Bossavit, Approximation régularisée d'un problème aux limites non homogène. Séminaire J.-L. Lions 12 (Avril 1969).

[7] F. Brezzi and M. Fortin, Mixed and Hybrid Finite Element Methods. Springer-Verlag (1991).

[8] P. Colli Franzoni, Approssimazione mediante il metodo de penalizazione de problemi misti di Dirichlet-Neumann per operatori lineari ellittici del secondo ordine. Boll. Un. Mat. Ital. A (7) 4 (1973) 229-250.

[9] P. Colli Franzoni, Approximation of optimal control problems of systems described by boundary value mixed problems of Dirichlet-Neumann type, in 5th IFIP Conference on Optimization Techniques. Springer, Berlin, Lecture Notes in Computer Science 3 (1973) 152-162.

[10] M. Costabel and M. Dauge, A singularly perturbed mixed boundary value problem. Commun. Partial Differential Equations 21 1919-1949 (1996).

[11] M. Dauge, Elliptic boundary value problems on corner domains. Smoothness and asymptotics of solutions. Springer-Verlag, Lecture Notes in Math. 1341 (1988).

[12] P. Grisvard, Singularities in boundary value problems. Masson (1992).

[13] L.S. Hou and S.S. Ravindran, A penalized Neumann control approach for solving an optimal Dirichlet control problem for the Navier-Stokes equations. SIAM J. Control Optim. 20 (1998) 1795-1814.

[14] L.S. Hou and S.S. Ravindran, Numerical approximation of optimal flow control problems by a penalty method: error estimates and numerical results. SIAM J. Sci. Comput. 20 (1999) 1753-1777.

[15] A. Kirsch, The Robin problem for the Helmholtz equation as a singular perturbation problem. Numer. Funct. Anal. Optim. 8 (1985) 1-20.

[16] I. Lasiecka and J. Sokolowski, Semidiscrete approximation of hyperbolic boundary value problem with nonhomogeneous Dirichlet boundary conditions. SIAM J. Math. Anal. 20 (1989) 1366-1387.

[17] J.-L. Lions, Contrôle optimal de systèmes gouvernés par des équations aux dérivées partielles. Dunod (1968).

[18] J.-L. Lions and E. Magenes, Problèmes aux limites non homogènes et applications, Vols. 1 and 2. Dunod, Paris (1968).

[19] T. Masrour, Contrôlabilité et observabilité des sytèmes distribués, problèmes et méthodes. Thesis, École Nationale des Ponts et Chaussées. Paris (1995). 